\title{
Relation entre la richesse du sol en phosphore et la concentration en phosphore de l'eau de drainage dans deux agro-écosystèmes Relationship between soil Phosphorus content and Phosphorus
concentration in drainage water in two agroecosystems
}

\author{
R. R. Simard et S. Beauchemin
}

Volume 15, numéro hors-série, 2002

URI : https://id.erudit.org/iderudit/705489ar

DOI : https://doi.org/10.7202/705489ar

\section{Aller au sommaire du numéro}

\section{Éditeur(s)}

Université du Québec - INRS-Eau, Terre et Environnement (INRS-ETE)

ISSN

0992-7158 (imprimé)

1718-8598 (numérique)

\section{Découvrir la revue}

Citer cet article

Simard, R. R. \& Beauchemin, S. (2002). Relation entre la richesse du sol en phosphore et la concentration en phosphore de l'eau de drainage dans deux agro-écosystèmes. Revue des sciences de l'eau / Journal of Water Science, 15, 109-120. https://doi.org/10.7202/705489ar

\section{Résumé de l'article}

Des concentrations en P excédant le seuil d'eutrophisation sont fréquemment mesurées dans l'eau des affluents du fleuve Saint-Laurent au Québec, Canada. Un enrichissement excessif en P des sols agricoles en serait la source. Une norme relative à la saturation en $\mathrm{P}$ des sols a été proposée comme critère de risque de contamination en $P$ des eaux de surface. L'objectif de ce travail est d'étudier le lien entre la richesse en $\mathrm{P}$ du sol et la teneur en P de l'eau de drainage dans deux agro-écosystèmes du Québec. Le bassin versant de la rivière Boyer (BVB), dominé par des sols en pente, une forte densité animale et des productions fourragères et les Basses Terres de Montréal (BTM), dominées par des sols plats utilisés pour la production intensive de maïs et une faible densité animale sont étudiés. Le degré de saturation en P des sols du BVB est de 8 à $10 \%$ alors que celui des BTM dépasse très souvent $15 \%$. Le pool du P organique est plus faible dans les sols des BTM que dans le BVB. La concentration moyenne en $\mathrm{P}$ de l'eau de drainage est plus élevée dans les sols du BVB $\left(171 \mu \mathrm{g} \mathrm{L}{ }^{-1}\right)$ que dans ceux des BTM $\left(98 \mu \mathrm{g} \mathrm{L}^{-1}\right)$. Elle est corrélée à la teneur en $\mathrm{P}$ extrait à l'oxalate des sols argileux et à la teneur en P soluble dans l'eau ou à l'index de sorption en $\mathrm{P}$ des sols grossiers. Ces relations sont plus étroites pour la couche $0-5 \mathrm{~cm}$ de sol que pour les couches plus profondes. Les résultats de cette étude démontrent qu'il est difficile de prédire la concentration en P de l'eau de drainage de sols contrastants. Regrouper les sols selon leur texture améliore la précision de la prédiction de la teneur en P de l'eau de drainage à partir de leurs propriétés. 


\title{
Relation entre la richesse du sol en phosphore et la concentration en phosphore de l'eau de drainage dans deux agro-écosystèmes
}

\author{
Relationship between soil Phosphorus content \\ and Phosphorus concentration in drainage water \\ in two agroecosystems
}

R.R. SIMARD ${ }^{1,2}$, S. BEAUCHEMIN ${ }^{3}$ *

\section{SUMMARY}

Concentrations of $P$ higher than the recognised threshold for eutrophication are often measured in the tributaries of the St. Lawrence river, Quebec, Canada. An excessive $P$ enrichment of agricultural soils was identified as the potential cause of this phenomenon. This enrichment results in a decrease in the $P$ sorption capacity of soils and an increased risk of $P$ contamination of surface waters. A norm based on soil P saturation degree (DSPS) was proposed as an agro-environmental criterion to reduce this risk. Relationships between DSPS and other soil $P$ attributes and surface runoff $P$ concentration have been reported in the literature but not for tile-drainage water. The objective of this work is to study the relationship between soil $\mathbf{P}$ attributes and drainage water $P$ in two agro-ecosystems of Quebec.

The Boyer watershed (BW), which is dominated by soils with significant slopes, a high animal density and forage production, and the Montreal lowlands (ML), dominated by flat soils, low animal density and mostly used for corn (Zea mays L.), were studied. The A, B and $C$ horizons from soils from the BW were sampled at three locations in tile-drained fields from farms in surplus or not of manure $\mathbf{N}$ in regard to crop needs. Soils were sampled in the ML according to a gradient in clay content with the same procedure. Soils were characterised for their $\mathrm{pH}$, particle size distribution, Mehlich 3extractable $P\left(P_{m 3}\right)$ and $A l$, water-soluble $P\left(P_{w}\right)$, organic $P(P o)$ and oxalate-

1. Soils Department, University of Manitoba, Winnipeg, Manitoba, R3T 2N2.

2. Agriculture et agroalimentaire Canada, Centre de recherches sur les sols et les grandes cultures, 2560 Hochelaga, Sainte-Foy, Qc, Canada, G1V2J3.

3. Natural Ressources Canada, 555 Booth, Ottawa, ON, Canada, KIA OGI.

* Correspondance. E-mail : sbeauche@nrcan.gc.ca

** Les commentaires seront reçus jusqu'au 30 avril 2003. 
extractable $\mathrm{P}\left(\mathrm{P}_{\mathrm{ox}}\right), \mathrm{Al}\left(\mathrm{Al}_{\mathrm{ox}}\right)$ and $\mathrm{Fe}\left(\mathrm{Fe}_{\mathrm{ox}}\right)$ contents. Grab tile-drainage samples were taken in triplicate and characterised for total (TP), particulate (PP) and dissolved reactive $P$ (RP) and unreactive $P$ (UP).

The soils of the $B W$ are more acidic and have higher $P$ retention capacities than ML soils. The $P_{w}$ content of $B W$ soils is lower than in ML ones in spite of comparable $\mathrm{P}_{\mathrm{m3}}$ contents. The DSPS $\left(\mathrm{P}_{\mathrm{ox}} / \mathrm{Al}_{\mathrm{ox}}+\mathrm{Fe}_{\mathrm{ox}}\right)$ of $\mathrm{BW}$ soils is moderate $(8$ to $10 \%$ ) whereas DSPS in ML soils is often $>15 \%$. The soil $P$ organic pool is much smaller in the ML soils than in those from BW, probably because of more frequent tillage and lower manure $C$ inputs.

The average $P$ concentration (TP) in drainage water was higher in soils from the BW $\left(171 \mu \mathrm{g} \cdot \mathrm{L}^{-1}\right)$ than from ML soils $\left(98 \mu \mathrm{g} \cdot \mathrm{L}^{-1}\right)$. The PP was the main $P$ fraction in drainage water from the two ecosystems. The RP was on average 44\% of TP whereas UP was much less. The TP concentration in drainage waters was higher than $0.03 \mathrm{mg} \cdot \mathrm{L}^{-1}$ in most cases. The TP concentration was higher in tile-drainage waters from the BW than in the ML even though comparable $P_{m 3}$ and lower DSPS were found in the BW than in ML soils. These results suggest that other criteria should be included in the prediction of the risk of $P$ contamination of drainage waters. The results of the present study indicate that agricultural practices (crop species, manure inputs, tillage type and frequency) could have a greater influence than soil $P$ status on the TP concentration in tile-drainage waters.

A linear correlation analysis between the logarithm of the TP $(T P+0.5)$ and that of the different soil $P$ attributes indicated that TP was related to the $P$ extracted by oxalate from clay soils and by water in coarse-textured soils. These relationships were closer in the $0-5 \mathrm{~cm}$ soil layer than in deeper strata. Multiple regression analysis between the logarithms of drainage water $P$ concentrations and soil attributes revealed that, when soils were grouped by texture, the prediction of $T P$ was much more accurate than when all soils were considered. The present regulatory approach of assessing the risk of water $P$ contamination by using the soil $P_{m 3}$ and DSPS only was inadequate in the two considered agroecosystems.

The results of this study indicate that the prediction of the drainage water $P$ concentration with $P$ attributes from contrasting soils is difficult. Grouping soils by texture improves the prediction of $P_{t}$ from soil $P$ attributes.

Key-words: phosphorus, drainage water, $P$ index, $P$ saturation degree, gleysols.

\section{RÉSUMÉ}

Des concentrations en $P$ excédant le seuil d'eutrophisation sont fréquemment mesurées dans l'eau des affluents du fleuve Saint-Laurent au Québec, Canada. Un enrichissement excessif en $P$ des sols agricoles en serait la source. Une norme relative à la saturation en $\mathbf{P}$ des sols a été proposée comme critère de risque de contamination en $P$ des eaux de surface. L'objectif de ce travail est d'étudier le lien entre la richesse en $P$ du sol et la teneur en $P$ de l'eau de drainage dans deux agro-écosystèmes du Québec. Le bassin versant de la rivière Boyer (BVB), dominé par des sols en pente, une forte densité animale et des productions fourragères et les Basses Terres de Montréal (BTM), dominées par des sols plats utilisés pour la production intensive de maïs et une faible densité animale sont étudiés. Le degré de saturation en $P$ des sols du BVB est de 8 à $10 \%$ alors que celui des BTM dépasse très souvent $15 \%$. Le pool du $P$ organique est plus faible dans les sols des BTM que dans le $B$ BB. La concentration moyenne en $P$ de l'eau de drainage est plus élevée dans les sols du BVB $\left(171 \mu \mathrm{g} \cdot \mathrm{L}^{-1}\right)$ que dans ceux des BTM $\left(98 \mu \mathrm{g} \cdot \mathrm{L}^{-1}\right)$. Elle est corrélée à la teneur en $P$ extrait à l'oxalate dans les sols argileux et à la teneur en $P$ soluble dans l'eau ou à l'index de sorption en $P$ dans les sols grossiers. Ces relations sont plus étroites pour la couche $0-5 \mathrm{~cm}$ de sol que pour 
les couches plus profondes. Les résultats de cette étude démontrent qu'il est difficile de prédire la concentration en $P$ de l'eau de drainage de sols contrastants. Regrouper les sols selon leur texture améliore la précision de la prédiction de la teneur en $\mathbf{P}$ de l'eau de drainage à partir de leurs propriétés.

Mots clés : phosphore, eau de drainage, index de $P$, degré de saturation en $P$, gleysols.

\section{1 - INTRODUCTION}

Des concentrations élevées de $P$ sont fréquemment retrouvées dans les affluents du Saint-Laurent au Québec (ministère de l'Environnement du Québec, 1993). Ce $P$ aurait pour origine les sols agricoles (PAINCHAUD, 1997). Les sols des zones d'élevage intensif sont fortement enrichis en P (SIMARD et al., 1995 ; SIMARD et al., 1998). C'est également le cas de certains sols de grandes cultures des basses terres de Montréal (BEAUCHEMIN et SIMARD, 2000). Cet enrichissement se traduit par une baisse significative de leur capacité à retenir le $P$ (BEAUCHEMIN et al., 1996). Le $P$ ajouté sous forme d'engrais minéral ou de déjections animales a donc un risque plus élevé d'atteindre les cours d'eaux.

Le ruissellement et l'érosion sont identifiés comme principaux modes de transport du $\mathrm{P}$ des sols vers les eaux de surface (Ministère de l'Environnement du Québec, 1993 ; SHARPLEY et al., 1995). Cependant, les sols à texture grossière et les sols argileux peuvent transférer des quantités importantes de $\mathrm{P}$ via l'eau de drainage (BEAUCHEMIN et al., 1998), particulièrement si ceux-ci sont propices à l'infiltration préférentielle (SIMARD et al., 2000a).

Afin de réduire la pollution diffuse en $P$ causée par l'agriculture, le ministère de l'Environnement du Québec (1998) a produit une norme P basée sur le principe de degré de saturation en $\mathrm{P}$ de la couche de labour des sols. Cette norme est basée sur le rapport des quantités de $P$ et d'Al extraites par le réactif Mehlich 3 (GIROUX et TRAN, 1996 ; KHIARI et al., 2000). Cette approche est pratique puisqu'elle permet d'utiliser les données archivées et les analyses de sol de routine (BEAUCHEMIN et SIMARD, 1999). Bien que l'utilité de ce ratio pour prédire la teneur en $P$ de l'eau de ruissellement ait été démontrée (POTE et al, 1999), cette approche n'a pas été évaluée comme critère de prédiction de la qualité de l'eau de drainage. L'objectif de cette étude est de comparer la relation entre la richesse en $\mathrm{P}$ du sol et la concentration en $\mathrm{P}$ de l'eau de drainage dans deux agro-écosystèmes du Québec.

\section{2 - MATÉRIAUX ET MÉTHODES}

Cette étude touche deux agro-écosystèmes : le bassin versant de la rivière Boyer (BVB) et les basses terres de Montréal (BTM). Le BVB, zone d'élevage intensif, est caractérisé par des sols à forte pente (2 à $15 \%$ ). Les plantes four- 
ragères occupent plus de $50 \%$ du territoire. Les BTM sont caractérisées par des sols plats, une faible densité animale et une production centrée sur le maïs grain et le soja (Glycine max [L.], Merr.).

Les sols des BTM ont été échantillonnés en automne 1994 selon un gradient de teneur en argile (BEAUCHEMIN et SIMARD, 2000). Les sols du BVB ont été prélevés à l'automne 1996 dans trois systèmes de production :

1. laitier en déficit de fumier par rapport aux besoins en $\mathrm{N}$ des cultures ;

2. porcin en excédent de lisier ;

3. mixte, porcin et laitier, en excédent de déjections (SIMARD et al., 1998).

Brièvement, les horizons génétiques $A, B$ et $C$ ont été échantillonnés sur trois sites séparés d'environ $10 \mathrm{~m}$. Les échantillons ont été séchés à l'air et tamisés à $<2 \mathrm{~mm}$.

Les échantillons de sol ont été caractérisés par leur $\mathrm{pH}$ (ratio sol : eau de 1:2). Les teneurs en $P$ et Al extraits au Mehlich $3\left(P_{m 3}, A I_{m 3}\right)$ ont été déterminées selon TRAN et SIMARD (1993). Les teneurs en P, Al et Fe solubles dans la solution d'oxalate acide d'ammonium $\left(\mathrm{P}_{\mathrm{ox}}, \mathrm{Al}_{\mathrm{ox}}, \mathrm{Fe}_{\mathrm{ox}}\right)$ ont été déterminées par la méthode décrite par ROSS et WANG (1993). La concentration totale en $P$ dans ces extraits a été mesurée après digestion acide selon la méthode de ROWLAND et GRIMSHAW (1995). La teneur en $P$ organique des sols $\left(P_{0}\right)$ a été déterminée après extraction par une solution $\mathrm{NaOH} 0,025 \mathrm{M}$ et $\mathrm{Na}_{2}$ EDTA 0,05 $\mathrm{M}$ (BOWMAN et MOIR, 1993). La teneur en $P$ total des sols a été déterminée par digestion dans le $\mathrm{HClO}_{4}$ (OLSEN et SOMMERS, 1982). La teneur en $\mathrm{P}$ soluble à l'eau $\left(\mathrm{P}_{\mathrm{w}}\right) \mathrm{a}$ été déterminée selon une méthode dérivée de SISSINGH (1971) et décrite dans BEAUCHEMIN et SIMARD (2000). L'indice de sorption du $P\left(P_{s i}\right)$ a été déterminé en utilisant la méthode de BEAUCHEMIN et SIMARD (2000). La teneur en $P$ des extraits a été déterminée par colorimétrie selon la méthode de MURPHY et RILEY (1962). Les teneurs en Al et Fe des filtrats ont été déterminées par spectroscopie d'absorption atomique (Perkin Elmer, 3003, Überlingen, ALL). Le P adsorbé sur membranes d'échange anioniques in situ $\left(P_{\text {res }}\right)$ a été déterminé par la méthode décrite dans SIMARD et al. (2000b).

L'eau de drainage a été recueillie in situ en triplicata à la sortie des drains agricoles. Les échantillons ont été congelés jusqu'à l'analyse. L'eau a été filtrée sur Millipore 0,45 $\mu \mathrm{m}$. Les eaux brutes et filtrées ont été analysées selon les méthodologies décrites dans BEAUCHEMIN et al. (1998). Les données de composition de l'eau de drainage $(x)$ ont été transformées log $(x+0,5)$ afin d'améliorer la normalité de leur distribution avant analyses de corrélation et de régression multiple (SAS Institute, 1990).

\section{3 - RÉSULTATS ET DISCUSSION}

\section{1 État en $\mathbf{P}$ des sols}

Le profil des sols du BVB est plus acide que celui des BTM (tableau 1). La teneur en $P_{w}$ des sols du BVB est inférieure à celle des BTM en dépit de 
Tableau 1 Caractéristiques moyennes des sols des deux agro-écosystèmes à l'étude.

Table 1 Mean characteristics of soils from the two agro-ecosystems under study.

\begin{tabular}{|c|c|c|c|c|c|c|}
\hline \multirow{2}{*}{ Paramètre } & \multicolumn{3}{|c|}{ Bassin versant de la Boyer } & \multicolumn{3}{|c|}{ Basses Terres de Montréal } \\
\hline & \multicolumn{3}{|c|}{ Horizon } & \multicolumn{3}{|c|}{ Horizon } \\
\hline & A & B & C & A & B & $\mathrm{C}$ \\
\hline & $\mathrm{n}=27$ & $\mathrm{n}=27$ & $\mathrm{n}=27$ & $n=27$ & $n=27$ & $\mathrm{n}=27$ \\
\hline $\mathrm{pH}$ & $5,6 \pm 0,4$ & $5,8 \pm 0,6$ & $5,8 \pm 0,6$ & $7,2 \pm 0,6$ & $7,4 \pm 0,6$ & $7,5 \pm 0,5$ \\
\hline $\mathrm{P}_{\mathrm{w}}\left(\mathrm{mg} \cdot \mathrm{kg}^{-1}\right)$ & $1,70 \pm 1,56$ & $0,77 \pm 0,78$ & $0,77 \pm 0,81$ & $11,2 \pm 8,0$ & $2,1 \pm 2,5$ & $1,8 \pm 1,9$ \\
\hline$P_{0 x}\left(\mathrm{mmol} \cdot \mathrm{kg}^{-1}\right)$ & $20 \pm 12$ & $17 \pm 13$ & $14,7 \pm 7,9$ & $17,7 \pm 10,7$ & $11,9 \pm 8,6$ & $12,9 \pm 7,2$ \\
\hline $\mathrm{Fe}_{0 \mathrm{x}}\left(\mathrm{mmol} \cdot \mathrm{kg}^{-1}\right)$ & $107 \pm 45$ & $101 \pm 31$ & $96 \pm 38$ & $88 \pm 216$ & $63 \pm 89$ & $68 \pm 94$ \\
\hline $\mathrm{Al}_{\mathrm{ox}}\left(\mathrm{mmol} \cdot \mathrm{kg}^{-1}\right)$ & $122 \pm 46$ & $119 \pm 47$ & $104 \pm 42$ & $66 \pm 41$ & $66 \pm 44$ & $68 \pm 37$ \\
\hline$P_{m 3}\left(m g \cdot k^{-1}\right)$ & 94 & 32 & 18 & $91 \pm 61$ & $14 \pm 18$ & $8 \pm 9$ \\
\hline $\mathrm{Al}_{\mathrm{m} 3}\left(\mathrm{mg} \cdot \mathrm{kg}^{-1}\right)$ & ND & ND & ND & $813 \pm 324$ & $800 \pm 367$ & $835 \pm 384$ \\
\hline$P_{0}\left(m g \cdot k g^{-1}\right)$ & $522 \pm 380$ & $318 \pm 227$ & $258 \pm 328$ & $152 \pm 123$ & $45 \pm 68$ & $25 \pm 37$ \\
\hline $\mathrm{P}_{\mathrm{si}}$ & $350 \pm 187$ & $379 \pm 171$ & $258 \pm 58$ & $220 \pm 137$ & $264 \pm 164$ & $304 \pm 165$ \\
\hline $\mathrm{P}_{\text {res }}\left(\mathrm{mg} \cdot \mathrm{kg}^{-1}\right)$ & ND & ND & ND & $93 \pm 39$ & $82 \pm 42$ & $83 \pm 39$ \\
\hline$P$ total $\left(\mathrm{mg} \cdot \mathrm{kg}^{-1}\right)$ & $1009 \pm 376$ & $649 \pm 306$ & $460 \pm 362$ & $946 \pm 276$ & $703 \pm 199$ & $696 \pm 166$ \\
\hline $\operatorname{DSPS}_{\mathrm{m} 3}(\%)$ & ND & ND & ND & $14,0 \pm 10,1$ & $2,1 \pm 2,9$ & $\uparrow, 1 \pm 1,1$ \\
\hline $\operatorname{DSPS}_{0 x}(\%)$ & $9,1 \pm 4,7$ & $7,7 \pm 5,6$ & $8,0 \pm 5,1$ & $16,3 \pm 7,8$ & $10,3 \pm 4,5$ & $10,3 \pm 3,7$ \\
\hline
\end{tabular}

$\mathrm{P}_{\mathrm{w}}: \mathrm{P}$ soluble à l'eau ; $\mathrm{P}_{\mathrm{ox}}: \mathrm{P}$ soluble à l'oxalate acide d'ammonium; $\mathrm{Fe}_{\mathrm{ox}}$ : Fe soluble à l'oxalate acide d'ammonium; $\mathrm{Al}_{\mathrm{ox}}: A$ s soluble à l'oxalate acide d'ammonium; $P_{\mathrm{m}_{3}}: P$ soluble dans la solution de Mehlich III $; \mathrm{Al}_{\mathrm{m} 3}$ : Al soluble dans la solution de Mehlich III $; \mathrm{P}_{0}: \mathrm{P}$ organique $; \mathrm{P}_{\mathrm{si}}$ : index de sorption du $P ; P_{\text {res }}: P$ extrait par une résine d'échange; $D S S_{33}:$ degré de saturation en $P$ du sol tel que déterminé dans la solution de Mehlich III ; DSPS $_{0 x}$ : degré de saturation en $P$ du sol tel que déterminé dans la solution d'oxalate acide d'ammonium ; ND : non-déterminé.

valeurs comparables de $P_{m 3}$. Cette observation peut être reliée aux valeurs plus élevées en $\mathrm{Fe}_{\mathrm{ox}}$ et $\mathrm{Al}_{\mathrm{ox}}$ dans le $\mathrm{BVB}$, suggérant une plus forte capacité de rétention en $P$ des sols du BVB que dans ceux des BTM. Les valeurs moyennes de $P_{m 3}$ des horizons A permettent de classer la fertilité en $P$ des sols des deux agro-écosystèmes comme élevée (GIROUX et TRAN, 1996). La teneur en $P$ organique est beaucoup plus faible dans les BTM que dans le BVB en dépit de teneurs plus élevées en $P$ total dans les horizons $B$ et $C$. Ces valeurs plus faibles en $\mathrm{P}$ organique pourraient être reliées à un bilan en $\mathrm{C}$ moins excédentaire dans les sols des BTM que dans ceux du BVB (ZHENG et al., 2001). De plus, un labour moins fréquent des sols du BVB réduit la minéralisation du $P$ organique. Les valeurs de P organique du BVB sont encore plus élevées que celles rapportées pour le bassin versant de la Beaurivage (SIMARD et al., 1995) ou celles rapportées par TRAN et N'DAYEGAMIYE (1995) pour un sol Le Bras ayant reçu des apports importants et répétés de fumier solide de bovins laitiers. Le degré de saturation en P du sol (DSPS ${ }_{\text {ox }}$ ) est moindre dans le BVB que dans les BTM (tableau 1) en raison de teneurs plus élevées en $\mathrm{Fe}_{\mathrm{ox}}+\mathrm{Al}_{\mathrm{ox}}$. De 
même, l'index de sorption du $P$ est plus élevé dans les horizons $A$ et $B$ des sols du BVB que dans ceux des BTM. Les sols du BVB sont davantage podzolisés, ce qui accentue leur capacité à retenir le $P$ comparé aux gleysols neutres à calcaires des BTM (BEAUCHEMIN et SIMARD, 1999 ; LECLERC et al., 2001). Bien que le DSPS $_{\text {ox }}$ des BTM soit plus élevé que celui du BVB, les teneurs en $P$ sorbé $\left(P_{o x}\right)$ des sols du BVB sont plus élevées. Cela suggère un historique légèrement plus important d'apports en P à ces sols qu'à ceux des BTM.

\subsection{Composition de l'eau de drainage}

Dans le BVB, le $P$ réactif dissout dans les 27 échantillons de l'eau de drainage a varié entre 1 et $165 \mu \mathrm{g} \cdot \mathrm{L}^{-1}$ et constituait, en moyenne, $44 \%$ du $\mathrm{P}_{\mathrm{t}}$ (tableau 2). Cette très grande variation de la teneur en $P$ était reliée, comme nous le verrons plus loin, à la grande diversité des teneurs en $P$ des sols étudiés. Le $P$ non-réactif dissous était beaucoup moins important (moyenne de $12 \mu \mathrm{g} \cdot \mathrm{L}^{-1}$ ). Cette fraction non-réactive est souvent associée au $P$ organique dissous (BEAUCHEMIN et al., 1998). Le $P$ particulaire constituait la fraction principale du $P$ de l'eau de drainage (moyenne $88 \mu \mathrm{g} \cdot \mathrm{L}^{-1}$ ). La teneur moyenne en $P_{t}$ $\left(171 \mu \mathrm{g} \cdot \mathrm{L}^{-1}\right)$ dépassait la norme proposée pour les eaux de surface (Ministère de l'Environnement du Québec, 1993). En fait, cette norme était dépassée dans 8 des 9 fermes à l'étude (SIMARD et al., 1998).

Tableau 2 Formes de phosphore dans l'eau de drainage des sols des deux agro-écosystèmes.

Table 2 Forms of phosphorus in drainage water from soils of the two agroecosystems.

\begin{tabular}{|c|c|c|c|c|c|c|}
\hline \multirow{2}{*}{$\begin{array}{c}\text { Forme } \\
\text { de P }\end{array}$} & \multicolumn{3}{|c|}{$\begin{array}{c}\text { Bassin versani de la Boyer } \\
\mathbf{n = 2 7}\end{array}$} & \multicolumn{3}{c|}{$\begin{array}{c}\text { Basses Terres de Montréal } \\
\mathbf{n = 2 7}\end{array}$} \\
\cline { 2 - 7 } & minimum & maximum & moyenne & minimum & maximum & moyenne \\
\hline PRD $\left(\mu \mathrm{g} \cdot \mathrm{L}^{-1}\right)$ & 1 & 165 & 71 & 5 & 665 & 40 \\
$\mathrm{PND}\left(\mu \mathrm{g} \cdot \mathrm{L}^{-1}\right)$ & $<1$ & 49 & 12 & $<1$ & 84 & 13 \\
$\mathrm{PP}\left(\mu \mathrm{g} \cdot \mathrm{L}^{-1}\right)$ & $<1$ & 158 & 88 & $<1$ & 432 & 45 \\
$\mathrm{P}_{\mathrm{t}}\left(\mu \mathrm{g} \cdot \mathrm{L}^{-1}\right)$ & 9 & 273 & 171 & 11 & 1105 & 98 \\
\hline
\end{tabular}

PRD : phosphore dissous $(<0,45 \mu \mathrm{m})$ réactif au molybdate ; PND phosphore dissous $(<0,45 \mu \mathrm{m})$ non-réactif au molybdate; $\mathrm{PP}$ : phosphore particulaire $; P_{t}:$ phosphore total.

Dans les BTM, la teneur en $\mathrm{P}$ réactif dissous dans l'eau de drainage a montré une variation plus importante que celle du BVB; cependant la teneur moyenne y est inférieure $\left(40 \mu \mathrm{g} \cdot \mathrm{L}^{-1}\right)$. La proportion de $\mathrm{P}$ sous forme de $\mathrm{P}$ réactif dissous est comparable à celle de l'eau de drainage du BVB (41 vs $42 \%$ ). La teneur moyenne en $P$ non-réactif dissous est également comparable à celle du BVB (tableau 2). La teneur moyenne en $P$ particulaire est de $45 \mu \mathrm{g} \cdot \mathrm{L}^{-1}$ et constitue également la forme principale de $\mathrm{P}$ dans l'eau de drainage pour cet agroécosystème. La teneur moyenne en $P_{t}\left(98 \mu \mathrm{g} \cdot \mathrm{L}^{-1}\right)$ est supérieure à la norme de qualité des eaux de surface. 
Les teneurs en $\mathrm{P}_{\mathrm{m} 3}$ et le DSPS $\mathrm{m}_{3}$ ont été retenues comme critères régissant les épandages de déjections animales et d'engrais minéraux sur les sols du Québec (Ministère de l'Environnement du Québec, 1998). Les teneurs en $P_{t}$ plus élevées dans les eaux de drainage du BVB que dans celles des BTM sont observées en dépit de teneurs en $\mathrm{P}_{\mathrm{m} 3}$ des sols comparables et d'un plus faible $\mathrm{DSPS}_{\mathrm{ox}}$. Cela suggère que d'autres critères doivent être inclus dans la prédiction du risque de contamination de l'eau. Parmi ceux-ci, on retrouve le risque d'écoulement préférentiel variant selon la texture du sol et la distance entre les drains (Ministère de l'Environnement du Québec, 1998). De plus, des critères relatifs au bilan en $\mathrm{P}$ du site, à la quantité totale de $\mathrm{P}$ ajoutée sous forme de déjections animales et d'engrais minéraux ainsi qu'au mode d'apport et d'incorporation des fumiers doivent être considérés (BOLINDER et al., 2000). Dans le cas du BVB, les déjections animales sont souvent épandues en surface, à l'automne, directement sur le feuillage des prairies, sans incorporation, ce qui augmente d'autant le risque de contamination en $P$ des eaux de surface. En effet, GANGBAZO et al. (1997) ainsi que SIMARD et al. (2000a) rapportent des pertes équivalentes en $\mathrm{P}$ dans l'eau de drainage sous prairie avec des apports de $\mathrm{P}$ sous forme de déjections animales trois fois moins importants que sous maïs. L'écoulement préférentiel a été proposé comme mode de transport du $\mathrm{P}$ pour expliquer la proportion importante de $P_{t}$ de l'eau de drainage sous forme de $P$ particulaire (BEAUCHEMIN et al., 1996 ; STAMM et al., 1998 ; SIMARD et al., 2000a). La pédogènese pourrait également être impliquée. HOODA et al. (1997) rapportent des pertes quatre fois plus faibles dans les podzols bien drainés que dans les gleysols. Les résultats de notre étude indiquent que les systèmes culturaux (type de culture, apports répétés de déjections animales, fréquence de labour) pourraient avoir une influence encore plus grande sur la teneur en $P$ de l'eau de drainage.

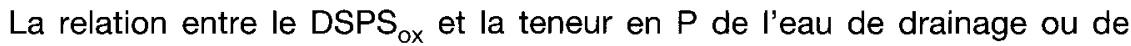
ruissellement ne serait pas unique mais influencée par d'autres facteurs tels la teneur en eau du sol dans les jours précédant l'échantillonnage de l'eau (BEAUCHEMIN et al., 1998 ; POTE et al., 1999). HOODA et al. (2000) indiquent que le $P_{\text {ox }}$ n'est pas un indice du $P$ qui peut être relâché de la phase solide dans la solution des sols non-calcaires.

\subsection{Relation entre le $\mathbf{P}$ du sol et la teneur en $\mathbf{P}_{\mathbf{t}}$ de l'eau de drainage}

Une analyse de corrélation linéaire entre le logarithme naturel de $\left(P_{t}+0,5\right)$ et celui des différents attributs du $\mathrm{P}$ du sol a été effectuée (tableau 3 ). Dans les BTM, le $P_{t}$ est plus étroitement corrélé à la teneur en $P_{o x}$ qu'aux teneurs en $P_{w}$, $P_{m 3}$ ou aux DSPS m $_{m 3}$ ou DSPS ${ }_{o x}$. Ces relations sont plus étroites pour la couche $0-5 \mathrm{~cm}$ ou l'horizon A que pour les horizons $B$ et $C$. De plus, cette relation n'est significative que pour les sols à texture fine ( $r=0,56$, données non-présentées). Dans le BVB, la teneur en $P_{t}$ de l'eau de drainage est étroitement liée au $P_{s i}$ et à la teneur en $P_{w}$ de la couche $0-5 \mathrm{~cm}$ de sol. La relation entre le $P_{s i}$ des autres couches de sol et le $P_{t}$ n'est pas significative. La relation entre $P_{t}$ et le $P_{o x}$ est beaucoup moins étroite dans les BTM. La plus faible proportion de sols argileux du BVB par rapport aux BTM pourrait en être la cause. En effet, la relation entre $P_{o x}$ et la teneur en $P_{t}$ est significative dans les sols argileux du BVB $(r=0,58$, couche $5-20 \mathrm{~cm}$ ) mais ne l'est pas pour les sols grossiers $(r<0,29$, données non-présentées). HOODA et al. (2000) rapportent que le $P_{\text {ox }}$ était la moins effi- 
cace parmi cinq méthodes d'extraction du $\mathrm{P}$ du sol pour prédire la quantité de $\mathrm{P}$ désorbé de 11 sols du Royaume-Uni. POTE et al. (1999) avaient rapporté un lien

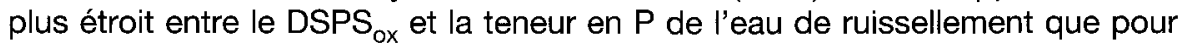
$P_{w}$, et $P_{m 3}$. Les résultats de notre étude indiquent que ce constat ne s'applique pas à l'eau de drainage des sols considérés. La relation entre le DSPS $_{o x}$ et $P_{t}$ n'est pas significative. Les faibles coefficients de corrélation observés entre les mesures de sol en $\mathrm{P}$ et la teneur en $\mathrm{P}_{t}$ de l'eau de drainage montrent bien la difficulté de prédire celle-ci dans des sols contrastants (HOODA et al., 1997 ; BEAUCHEMIN et al., 1998). Des facteurs de gestion tels le nombre de jours depuis la fertilisation peuvent avoir un effet plus grand que la richesse du sol sur le $\mathrm{P}_{\mathrm{t}}$ de l'eau de drainage (NASH et al., 2000).

Tableau 3 Coefficients de corrélation linéaire entre le logarithme de la teneur en phosphore total de l'eau de drainage $\left(P_{t}+0,5\right)$ et le logarithme de certains attributs en $\mathrm{P}$ des sols de deux agro-écosystèmes.

Table $3 \quad$ Linear correlation coefficients between the logarithm of the total $P$ concentration in drainage water $\left(P_{t}+0.5\right)$ and the logarithm of some $P$ attributes of soils from the two agroecosystems.

\begin{tabular}{|c|c|c|c|c|c|}
\hline \multirow{2}{*}{$\begin{array}{c}\text { Horizon/ } \\
\text { Couche } \\
n=27\end{array}$} & $\mathbf{P}_{\mathbf{w}}$ & $\mathbf{P}_{\mathrm{m} 3}$ & $P_{0 x}$ & DSPS $_{\mathbf{0 x}}$ & $\mathbf{P}_{\mathrm{si}}$ \\
\hline & \multicolumn{5}{|c|}{ Sols des Basses Terres de Montréal $n=27$} \\
\hline $0.5 \mathrm{~cm}$ & $0,27^{\star}$ & $0,12^{\mathrm{NS}}$ & $0,54^{* *}$ & $0,06^{\mathrm{NS}}$ & $0,34^{*}$ \\
\hline$A p$ & $0,25^{\mathrm{NS}}$ & 0,06 NS & $0,52^{*}$ & 0,09 NS & $0,33^{*}$ \\
\hline $\mathrm{Bg}$ & $0,31^{*}$ & $0,30^{*}$ & $0,46^{*}$ & $0,27 \mathrm{NS}$ & $0,24 \mathrm{NS}$ \\
\hline \multirow[t]{2}{*}{$\mathrm{Cg}$} & $0,39^{*}$ & $0,43^{*}$ & $0,35^{\mathrm{NS}}$ & $0,22^{N S}$ & $0,21^{N S}$ \\
\hline & \multicolumn{5}{|c|}{ Sols du bassin versant de la rivière Boyer $n=27$} \\
\hline $0-5 \mathrm{~cm}$ & $0,37^{*}$ & $0,32^{*}$ & $0,32^{*}$ & $-0,09 \mathrm{NS}$ & $0,30^{*}$ \\
\hline $5-20 \mathrm{~cm}$ & $0,33^{*}$ & 0,06 NS & $0,41^{*}$ & $-0,01^{N S}$ & $0,26^{\mathrm{NS}}$ \\
\hline $20-40 \mathrm{~cm}$ & $0,34^{*}$ & $-0,30^{\mathrm{NS}}$ & $0,10^{\mathrm{NS}}$ & 0,09 NS & $-0,01^{\mathrm{NS}}$ \\
\hline $40-60 \mathrm{~cm}$ & $0,34^{\star}$ & $-0,28^{N S}$ & $0,33^{*}$ & 0,16 NS & $0,31^{\mathrm{NS}}$ \\
\hline $60-80 \mathrm{~cm}$ & $0,32^{\star}$ & $-0,20^{\mathrm{NS}}$ & $0,12^{\mathrm{NS}}$ & $-0,15^{N S}$ & $0,00^{N S}$ \\
\hline
\end{tabular}

$n$ : nombre d'échantillons; $P_{w}: P$ soluble à l'eau $; P_{o x}: P$ soluble à l'oxalate acide d'ammonium; $\mathrm{P}_{\mathrm{m} 3}$ : $\mathrm{P}$ soluble dans la solution de Mehlich III ; DSPS ${ }_{0 x}$ : degré de saturation en $\mathrm{P}$ du sol tel que déterminé dans la solution d'oxalate acide d'ammonium ; $P_{\mathrm{si}}$ : index de sorption du $\mathrm{P}$; * significatif au niveau de probabilité $p<0,05 ;{ }^{* *}$ significatif à $p<0,01 ;$ NS : non-significatif.

\subsection{Prédiction de la teneur en $\mathbf{P}$ de l'eau de drainage à partir des analyses de sol}

Une analyse de régression multiple a été effectuée entre le logarithme naturel de la teneur en $P_{t}$ de l'eau de drainage et celui des attributs en $\mathrm{P}$ du sol. Cette analyse a été répétée en divisant les sols en deux groupes : texture fine $\left(>280 \mathrm{~g} \cdot \mathrm{kg}^{-1}\right.$ argile) ou grossière (LECLERC et al., 2001). 
La combinaison de la teneur en $\mathrm{P}_{\text {ox }}$ et de DSPS ${ }_{\text {ox }}$ de l'horizon A des sols, prédit le mieux la teneur en $P_{t}$ de l'eau de drainage des BTM (tableau 4). Cependant, la valeur du $\mathrm{R}^{2}$ est faible $(0,33)$. Dans le cas des sols à texture fine, le $R^{2}$ atteint $43 \%$ avec la combinaison de $P_{\text {ox }}$ et de $D S P S_{\text {ox. }}$ Le $R^{2}$ est plus faible dans les sols grossiers $\left(R^{2}<0,37\right)$. D'ailleurs, c'est la combinaison de la teneur en $\mathrm{Ca}$ échangeable et de $\mathrm{Fe}_{\mathrm{ox}}+\mathrm{Al}_{\text {ox }}$ qui donne les meilleurs résultats $\left(R^{2}=0,75\right.$; BEAUCHEMIN, 1996). II a été démontré que le regroupement des sols à texture fine et à substratum calcaire permettait d'avoir de meilleurs résultats (BEAUCHEMIN, 1996). L'étude de LECLERC et al. (2001) indique également que la texture et la réaction du sol $(\mathrm{pH})$ sont deux « facteurs » importants dans la prédiction du risque de désorption du $\mathrm{P}$ de la couche de labour des sols des BTM.

Tableau 4 Équations de régression prédisant la concentration en $\mathrm{P}$ total de l'eau de drainage à partir des propriétés de diverses couches de sols de deux agro-écosystèmes du Québec.

Table 4 Regression equations predicting the total $P$ concentration in drainage water from properties of different soil layers/horizons from two agroecosystems of Quebec.

\begin{tabular}{|c|c|c|}
\hline $\begin{array}{l}\text { Groupe de sols/ } \\
\text { couche ou horizon }\end{array}$ & Équation $Y=\log \left(P_{t}+0,5\right)$ & $\mathbf{R}^{2}$ \\
\hline & \multicolumn{2}{|l|}{ Sols des basses-terres de Montréal } \\
\hline $\begin{array}{l}\text { Tous les sols } / 0-5 \mathrm{~cm} \\
\mathrm{n}=27\end{array}$ & $Y=-1,291+0,267 \log \left(P_{0 X}\right)$ & 0,29 \\
\hline Tous les sols/Ap & $Y=-1,66+0,282 \log \left(P_{0 x}\right)+0,133 \log \left(\right.$ DSPS $\left._{0 x}\right)$ & 0,33 \\
\hline $\begin{array}{l}\text { Sols argileux } / 0-5 \mathrm{~cm} \\
\mathrm{n}=12\end{array}$ & $Y=-2,37+0,404 \log \left(P_{0 x}\right)+0,253 \log \left(\right.$ DSPS $\left._{0 x}\right)$ & 0,43 \\
\hline $\begin{array}{l}\text { Sols } n=\operatorname{argileux} / A p \\
n=12\end{array}$ & $Y=-2,09+0,391 \log \left(\mathrm{Pi}_{\mathrm{ox}}\right)+0,224 \log \left(\mathrm{DSPS}_{\mathrm{ox}}\right)$ & 0,39 \\
\hline $\begin{array}{l}\text { Sols grossiers/Ap } \\
n=15\end{array}$ & $Y=-0,59+0,012 \log \left(\mathrm{DSPS}_{\mathrm{m} 3}\right)-0,037 \log \left(\mathrm{Pi}_{\mathrm{ox}}\right)$ & 0,34 \\
\hline \multirow[t]{2}{*}{$\begin{array}{l}\text { Sols grossiers/Ap-Cg } \\
n=15\end{array}$} & $Y=-0,545-0,049 \log \left(P_{\text {res }}\right)+0,0212 \log \left(\mathrm{Fe}_{\mathrm{ox}}\right)$ & 0,42 \\
\hline & \multicolumn{2}{|l|}{ Sols du bassin versant de la rivière Boyer } \\
\hline $\begin{array}{l}\text { Tous les sols } / 0-5 \mathrm{~cm} \\
\mathrm{n}=27\end{array}$ & $Y=-0,432-0,061 \log \left(\mathrm{Fe}_{\mathrm{ox}}\right)+0,0936 \log \left(\left.\mathrm{A}\right|_{\mathrm{ox}}\right)$ & 0,33 \\
\hline $\begin{array}{l}\text { Sols argileux } / 5-20 \mathrm{~cm} \\
n=12\end{array}$ & $Y=2,416+0,221 \log \left(A I_{o x}\right)+0.0851 \log \left(P_{o X}\right)$ & 0,80 \\
\hline $\begin{array}{l}\text { Sols grossiers } / 5-20 \mathrm{~cm} \\
n=15\end{array}$ & $Y=-0,481-0,039 \log \left(P_{w}\right)+0,0292 \log \left(\left.A\right|_{o x}\right)$ & 0,63 \\
\hline
\end{tabular}

$\mathrm{n}:$ nombre d'échantillons considérés dans le modèle $; P_{t}: P$ total dans l'eau de drainage $\left(\mu \mathrm{g} \cdot \mathrm{L}^{-1}\right)$; $\mathrm{P}_{\text {ox }}$ : $\mathrm{P}$ soluble à l'oxalate acide d'ammonium $\left(\mathrm{mmol} \cdot \mathrm{kg}^{-1}\right) ; \mathrm{DSPS}_{0 \mathrm{x}}$ : degré de saturation en $\mathrm{P}$ du sol tel que déterminé dans la solution d'oxalate acide d'ammonium (\%) : $P_{w}: P$ soluble à l'eau $\left(\mathrm{mg} \cdot \mathrm{kg}^{-1}\right) ; \mathrm{Pi}_{\mathrm{ox}}: \mathrm{P}$ inorganique soluble à l'oxalate acide d'ammonium $\left(\mathrm{mmol} \cdot \mathrm{kg}^{-1}\right) ; \mathrm{DSPS}_{\mathrm{m}}$ : degré de saturation en $\mathrm{P}$ du sol tel que déterminé dans la solution de Mehlich III $(\%) ; \mathrm{P}_{\text {res }}$ : $\mathrm{P}$ sorbé sur les membranes de résine d'échange in situ $\left(\mu \mathrm{g} \cdot \mathrm{cm}^{-2}\right) ; \mathrm{Fe}_{0 x}: F e$ soluble à l'oxalate acide d'ammonium $\left(\mathrm{mmol} \cdot \mathrm{kg}^{-1}\right) ; \mathrm{Al}_{\mathrm{ox}}:$ Al soluble à l'oxalate acide d'ammonium $\left(\mathrm{mmol} \cdot \mathrm{kg}^{-1}\right)$. 
La prédiction de la teneur en $P_{t}$ de l'eau de drainage des sols du BVB à partir des données de sols est encore plus infructueuse $\left(R^{2}<0,33\right)$. Cependant, la combinaison $\mathrm{Al}_{\text {ox }}$ et $\mathrm{P}_{\text {ox }}$ de la couche $5-20 \mathrm{~cm}$ des sols argileux permet de prédire $80 \%$ de la variation de la teneur en $P_{t}$ de l'eau de drainage. Dans le cas des sols grossiers, la combinaison $\mathrm{Al}_{\mathrm{ox}}$ et $\mathrm{P}_{\mathrm{w}}$ de la couche $5-20 \mathrm{~cm}$ a le plus de succès $\left(R^{2}=0,63\right)$. Ces résultats suggèrent que la prédiction de la teneur en $P_{t}$ à partir d'analyses de sols très différents est une tâche difficile. Des regroupements basés sur la nature des sols (texture, nature du substratum) sont nécessaires afin d'améliorer la précision de l'estimation de $P_{t}$ (BEAUCHEMIN, 1996).

Plusieurs études récentes suggèrent l'utilisation d'une teneur limite à l'analyse de sol comme critère permettant de décrire le risque de contamination en $P$ des eaux de surface par les sols (HESKETH et BROOKES, 2000). Au Québec, une approche intégrant la teneur en $\mathrm{P}_{\mathrm{m} 3}$ et le DSPS $_{\mathrm{m} 3}$ a été retenue. L'utilisation de cette approche ne permet d'obtenir un diagnostic exact que dans $33 \%$ (9/27) des sols des BTM. Le risque est exagéré (teneur en $P_{t}$ inférieure à $30 \mu \mathrm{g} \cdot \mathrm{L}^{-1}$ ) dans 8 cas sur 27 et sous-estimé dans 10 autres cas. Dans le cas du BVB, le risque est adéquatement prédit dans 4 cas sur 9 et il est sous-estimé dans tous les autres cas. Ces résultats indiquent que l'approche d'estimation du risque basée sur la teneur de la couche de surface en $P_{m 3}$ et son DSPS ne convient que très peu pour prédire les pertes de $P$ dans l'eau de drainage. L'écoulement préférentiel doit être considéré (BEAUCHEMIN et al., 1998 ; SIMARD et al., 2000a).

\section{4 - CONCLUSIONS}

Cette étude confirme que certains sols des BTM et du BVB sont fortement enrichis en $\mathrm{P}$. Une grande proportion de ces sols peut transférer du P via l'eau de drainage aux eaux de surface (fossés) qui atteignent éventuellement les affluents du Saint-Laurent. Le lien statistique entre le $P_{m 3}$ et le $D S P S_{m 3}$ de l'horizon de surface des sols et la teneur en $P_{t}$ de l'eau de drainage qui s'écoule de ces sols est faible. La prédiction du risque de contamination en $P$ des eaux de surface à partir de ces deux indices devient donc très hasardeuse. II apparaît que des indices plus complexes incluant les modes de transport de l'eau et de gestion des sols devraient être adoptés pour mieux identifier les parcelles ayant un risque élevé de contaminer en $\mathrm{P}$ les eaux de surface. Cette étude confirme également l'avantage de regrouper les sols selon leur nature (texture, type de substratum) afin d'améliorer la prédiction de la teneur en $\mathrm{P}$ de l'eau de drainage à partir d'indices reliés à la richesse en $\mathrm{P}$ du sol. 


\section{RÉFÉRENCES BIBLIOGRAPHIQUES}

BEAUCHEMIN S., 1996. Évaluation du risque de lessivage du phosphore dans les sols agricoles. Th. Doct., INRS-Eau. $154 \mathrm{p}$.

BEAUCHEMIN S., SIMARD R.R., CLUIS D., 1996. Phosphorus sorption-desorption kinetics of soil under contrasting land uses. J. Environ. Qual., 25, 1317-1325.

BEAUCHEMIN S., SIMARD R.R., CLUIS D. 1998. Forms and concentration of phosphorus in drainage water of 27 tile-drained soils. J. Environ. Qual., 27, 721-728.

BEAUCHEMIN S., SIMARD R.R., 1999. Soil phosphorus saturation degree: Review of some indices and their suitability for $P$ management in Québec, Canada. Can. J. Soil Sci., 79, 615-625.

BEAUCHEMIN S., SIMARD R.R., 2000. Phosphorus status of intensively cropped soils of the St. Lawrence lowlands. Soil Sci. Soc. Am. J., 64, 659-670.

BOLINDER M.A., SIMARD R.R., BEAUCHEMIN S., MACDONALD K.B., 2000. Indicator of risk of water contamination by $P$ for soil landscape of Canada polygons using an indexing approach. Can. J. Soil Sci., 80, 153-163.

BOWMAN R.A., MOIR J.A., 1993. Basic EDTA extractant for soil organic phosphorus. Soil Sci. Soc. Am. J., 57, 15161518.

GANGBAZO G., PESANT A.R., BARNETT G.M., 1997. Effet de l'épandage des engrais minéraux et de grandes quantités de lisier de porc sur l'eau, le sol et les cultures. Ministère de l'Environnement du Québec, Ste-Foy, Québec, Rapport de recherches, $46 \mathrm{p}$.

GIROUX M., TRAN T.S., 1996. Critères agronomiques et environnementaux liés à la disponibilité, la solubilité et la saturation du phosphore des sols agricoles du Québec. Agrosol, 9, (2), 51-57.

HESKETH N., BROOKES P.C., 2000. Development of an indicator for risk of phosphorus leaching. J. Environ. Qual., 29, 105-110.

HOODA P.S., MOYNAGH M., SVOBODA I.F., THURLOW M., STEWART M., ANDERSON M.A., 1997. Soil and land use effects on phosphorus in six streams draining small agricultural catchments in Scotland. Soil Use Manage., 13, 196204.

HOODA P.S., RENDELL A.R., EDWARDS A.C., WITHERS P.J.A., AITKEN M.N., TRUESDALE V.W., 2000. Relating soil phosphorus indices to potential phosphorus release to water. J. Environ. Qual. 29, 1166-1171.

KHIARI L., PARENT L.E., PELLERIN A., ALIMI A.R.A., TREMBLAY C., SIMARD R.R., FORTIN J., 2000. An agri-environmental phosphorus saturation index for coarse-textured soils. J. Environ. Qual, 27, 1561-1567.

LECLERC M.L., NOLIN M.C., CLUIS D., SIMARD R.R., 2001. Grouping soils of the Montreal lowlands (Quebec) according to $\mathrm{P}$ sorption and desorption characteristics. Can. J. Soil Sci, 81, 71-83.

Ministère de l'Environnement du Québec, 1993. État de l'Environnement au Québec, 1992. Montréal, Guérin. 560 p.

Ministère de l'Environnement du Québec, 1998. Proposition de norme sur la fertilisation phosphatée au groupe de travail interministériel. Document de travail. Groupe technique sur la norme sur le phosphore, Québec, Qc, 64 p.

MURPHY J., RILEY J.P., 1962. A modified single solution method for the determination of phosphate in natural waters. Anal. Chim. Acta. 27, 31-36.

NASH D., HANNAH M., HALLIWELL D., MURDOCH C., 2000. Factors affecting phosphorus export from a pasture-based grazing system. J. Environ. Qual. 29, 1160-1166.

OLSEN S.R., SOMMERS L.E., 1982. Phosphorus In Methods of soil analysis. Part $2,2^{\text {nd }}$ ed., Agronomy $N^{\circ} 9, A m$. Soc. Agron., PAGE A.L. et al. (eds., 403-430.

PAINCHAUD J., 1997. Tendances de la qualité de l'eau des rivières du Québec, 1979-1994. Vecteur Environnement, 30, 43-50.

POTE D.H., DANIEL T.C., NICHOLS D.J., SHARPLEY A.N., MOORE P.A Jr., MILLER D.M., EDWARDS, D.R., 1999. Relationship between phosphorus levels in 
three Ultisols and phosphorus concentration in runoff. J. Environ. Qual., 28, 170-175.

ROSS G.J., WANG C., 1993. Extractable Al, $\mathrm{Fe}, \mathrm{Mn}$ and $\mathrm{Si}$. In: CARTER M.R. (ed.), Soil sampling and methods of analysis, 239-246.

ROWLAND A.P., GRIMSHAW H.M., 1985. A wet oxydation procedure suitable for total nitrogen and phosphorus in soil. Commun. Soil Sci. Plant Anal. 16, 551560.

SAS Institute, 1990. Statistics, $6^{e}$ edition, SAS Institute Inc. Cary, NC.

SHARPLEY A.N., HEDLEY M., SIBBESEN E., HILLBRICHT-ILKOWSKA A., HOUSE W.A., RYSZKOWSKI L., 1995. Phosphorus transfers from terrestrial to aquatic ecosystems. In: TIESSEN H. (ed.), Phosphorus in the global environment: transfers, cycles and management, 201-228.

SIMARD R.R., CLUIS D., GANGBAZO G., BEAUCHEMIN S., 1995. Phosphorus status of forest and agricultural soils from a watershed of high density. J. Environ. Qual., 24, 1010-1017.

SIMARD R.R., BEAUCHEMIN S, NOLIN M.C., 1998. Farming system type as a determinant factor in soil and water $\mathbf{P}$ enrichment in a concentrated livestock area. In: Proc. World Soil Sci. Cong., Montpelier, Fra., 20-28 Aug. 1998, Paper 607, [cd-rom].
SIMARD R.R., BEAUCHEMIN S., HAYGARTH P.M., 2000a. Potential for preferential pathways of phosphorus transport. J. Environ. Qual., 29, 97-105.

SIMARD R.R., CAMBOURIS A.N., NOLIN M.C., 2000b. Spatio-temporal variation of AEM-P in a corn field. I $n$ : Proc. $5^{\text {th }}$ Prec. Ag. Conf., 19-23 Juil. 2000, Minneapolis, [cd-rom].

SISSINGH H.A., 1971. Analytical procedure of the Pw-method, used for the assessment of the phosphate status of arable soils in the Netherlands. Plant Soil, 34 , 483-486.

STAMM C., FLÜHLER H., GÄCHTER R., LUENBERGER J., WUNDERLI W., 1998. Preferential transport of phosphorus in drained grassland soils. J. Environ. Qual. 27, 515-522.

TRAN T.S., SIMARD R.R., 1993. Mehlich IIIextractable elements. In: CARTER M.R. (ed.), Soil sampling and methods of analysis, 43-49.

TRAN T.S., N'DAYEGAMIYE A., 1995. Longterm effects of fertilizers and manure application on the forms and availability of soil phosphorus. Can. J. Soil Sci., 75, 281-285.

ZHENG Z., SIMARD R.R., LAFOND J., PARENT L.E., 2001. Phosphorus fractions of a humic gleysol as influenced by cropping systems and nutrient sources. Can. J. Soil Sci., 81, 175-183. 